
for Mechanical Engineering

\title{
Investigation of the fake reject cases with unqualified operational dimension based on the error compensation and tolerance compression
}

\begin{tabular}{|r|l|}
\hline Journal: & Transactions of the Canadian Society for Mechanical Engineering \\
\hline Manuscript ID & TCSME-2021-0005.R1 \\
\hline Manuscript Type: & Article \\
\hline Author: & 12 -Mar-2021 \\
\hline $\begin{array}{r}\text { Complete List of Authors: } \\
\text { Jiaozuo } \\
\text { Jiao, Feng; Henan Polytechnic University, } \\
\text { Feng, Wenhui; Huanghe Jiaotong University } \\
\text { Bie, Wenbo; Henan Polytechnic University } \\
\text { Chen, Fan; Pingdingshan University }\end{array}$ & $\begin{aligned} \text { Keywords: } \\
\text { fake reject, operational dimension, dimension chain, error compensation, } \\
\text { tolerance compression }\end{aligned}$ \\
\hline $\begin{array}{r}\text { Is the invited manuscript for } \\
\text { consideration in a Special } \\
\text { Issue? : }\end{array}$ & \begin{tabular}{l} 
Not applicable (regular submission) \\
\hline
\end{tabular} \\
\hline
\end{tabular}

\section{SCHOLARONE ${ }^{\text {M }}$ Manuscripts}




\title{
Investigation of the fake reject cases with unqualified operational dimension based on the error compensation and tolerance compression
}

\author{
Xiaosan $\mathrm{Ma}^{1,2}$, Feng Jiao ${ }^{1 *}$, Wenhui Feng ${ }^{2}$, Wenbo Bie ${ }^{1}$, Fan Chen ${ }^{3}$ \\ 1. School of Mechanical and Power Engineering, Henan Polytechnic University, Jiaozuo454000, Henan, China. \\ 2. College of Mechanical and Electronic Engineering, Huanghe Jiaotong University, Jiaozuo454950, Henan, China. \\ 3. School of Electrical and Mechanical Engineering, Pingdingshan University, Pingdingshan467000, Henan, China.
}

*Corresponding author

Feng Jiao

School of Mechanical and Power Engineering,

Henan Polytechnic University,

Henan, China

E-mail:mmaxiaosan@126.com

Tel: +8613625696613 


\begin{abstract}
A fake reject (FR) with unqualified operational dimension often occurs during part machining when the operational data do not coincide with the design ones. This may lead to unnecessary waste. A novel judgment and remedial measures for FR are investigated in this paper to enhance products' qualification rate. Firstly, the reasons for the FR occurrence are discussed based on the fact that the operational dimension tolerance calculated using the worst-case method of the process dimension chain calculation is too tight. Secondly, a novel judgment method of FR is proposed by calculating a new dimension chain. The operational dimension is treated as the concluding link. The actual deviations of the dimensions generated before the operational dimension generation are used to replace their upper and lower deviations. Finally, based on the error compensation relationship among the component links in the process dimension chain, a novel remedial measure of FR by compressing dimension tolerance in subsequent processing is proposed. The calculation flow of the dimension tolerance after tolerance compression is worked out. This study's results of this study are considered for instrumental in judging and processing FR in part machining.
\end{abstract}

Key words: fake reject; operational dimension; dimension chain; error compensation; tolerance compression; 


\section{Introduction}

During the processing of mechanical parts, the operational dimension and tolerance need to be calculated through the process dimension chain when the operational data do not coincide with the design data (Wade 1967; Zhang et al. 2012; Martin et al. 2015; Shah et al. 1998). Although the operational dimension is not directly marked in the drawing, it is a component link in the process dimension chain and is generated directly during machining. The concluding link of the process dimension chain is a design dimension (i.e., the blueprint dimension termed in some studies) and is marked directly in the design drawing. However, it can only be obtained indirectly during machining(Wade 1967; Zhang et al. 2012; Wang et al. 2016). During the actual processing, the operational dimension often exceeds its tolerance specification obtained by calculation. In contrast, the corresponding design dimension of the concluding link can still meet the design drawing requirement. In other words, under the condition that the operational dimension is not qualified, the part still is possibly qualified. These "possible" qualified parts are referred to as fake reject (FR) (Wang et al. 2014, 2019; Liu 2015), false waste product (Wang and Ren 2012; Hao et al. 2019; Chen 2011), or false junk (Hu and Zhang 2012). Given this, the appropriate remedial measures in subsequent processing often need to be taken to ensure that the design dimension is qualified.

In actual production, the operational dimension's disqualified situation will be passed to the corresponding design dimension obtained indirectly after the FR occurrence. If the remedial measures in the subsequent processing are overlooked, this will lead to real reject, i.e., part with an unqualified design dimension. Meanwhile, the emergence of FR is likely to cause misjudgment for qualified products, and it may increase the cost of product inspection (Li et al. 2011; Heling et al. 2016; Sfantsikopoulos 1990).

To reduce the rate of real reject and processing costs, extensive studies on FR have been carried out in the last decade(Wang et al. 2014, 2019; Liu 2015; Wang and Ren 2012; Hao et al. 2019; Chen 2011; Hu and Zhang 2012; Li 2005). Thus, Wang, et al. (2014; 2019), Wang and Ren(2012), and Hao et al. (2019) calculated the tolerance of the operational dimension for the FR based on the virtual tolerance theory. Virtual tolerance, which is tolerance with the lower deviation exceeding the upper one (Wang et al.2013, 2019; Wang and Ren 2012), has been introduced as an opposite concept to tolerance since tolerance restricts error while virtual tolerance compensates error. For a dimension with tolerance, the larger the range between upper and lower deviations and the larger the allowable error, the easier it will 
be to ensure the dimension. Regarding a dimension with virtual tolerance, the case is just the opposite(Wang and Ren 2012; Wang, et al. 2013): the corresponding dimension's tolerance value can be directly used as a negative value to calculate the dimension chain, especially the complex dimension chain, in the process of product design. Based on the concept of virtual tolerance, the tolerance, and the error compensation amount can be calculated simultaneously, leading to a simplified analysis and calculation process. Thus, the computer-aided design was also facilitated(Wang et al. 2013, 2014). Alternatively, Hu and Zhang(2012) introduced the improved worst-case method to calculate the process dimension chain for the FR's judgment. According to the error compensation of each link's dimension in the process dimension chain, Chen(2011) proposed an alternative method for judging the FR. The above studies introduced strong-generalty calculation methods to judge FR based on the process dimension chain. However, they failed to account for the effect of actual deviations (i.e., numerical values obtained by subtracting the nominal value from the actual value of a dimension) of other dimensions generated before the process dimension on the FR judgment. This deficiency deteriorated the accuracy of the FR's calculated operational dimension tolerance.

An attempt to mitigate this deficiency was made by Liu(2015), who put forward a formula of FR identification based on the actual dimension measurement method and converted it into a chart to facilitate FR's judgment in the production process. Li(2005) analyzed the FR's causes and adopted the re-inspection methods to distinguish FR from the real reject from the point of formation and measurement of the operational dimension during machining. Further, Li(2012) analyzed FR's causes from the aspects of operational dimension calculation, part machining, and dimension measurement and put forward the judgment method of FR and the improvement measures to avoid FR. From the standpoint of part machining and dimension measurement, these studies took account of the actual deviation of other dimensions, except for the operational dimension, which yielded the results close to the actual machining situations. However, they did not explore the operational dimension tolerance that could be used to identify FR from the dimension chain calculation standpoint. The determination steps of FR are more complicated.

Moreover, the above studies disregarded that the machining operation was usually not completed when the FR with an unqualified operational dimension occurred, and the FR could be remedied in subsequent processing. To the best of the authors' knowledge, no comprehensive investigations have been conducted to substantiate possible remedial measures. Therefore, a more in-depth analysis of the 
FR's remedial measures in the subsequent processing is quite topical.

In this paper, possible reasons for the generation of FR are analyzed based on the principle and characteristics of process dimension chain calculation. By taking the operational dimension as the concluding link, a new dimension chain is established. A new tolerance of the operational dimension is obtained through the calculation of this new dimension chain. Then by combining this new operational dimension tolerance and the actual deviations of the other dimensions in the dimension chain during machining, a new calculation and judgment method of FR is put forward. Finally, in view that the operational dimension is obtained during the part machining, a novel remedial measures on FR, which allow one to avoid processing FR into a real reject with an unqualified design dimension, are presented and substantiated.

The present study's flowchart is depicted in Fig. 1.

\section{Reasons for the fake reject occurrence}

\subsection{Analysis of the fake reject causes}

During the part machining, when the operational data do not coincide with the design data, it is necessary to calculate the operational dimension and tolerance using the process dimension chain. It is aimed to determine the nominal value $D_{O p e}$, the upper deviation $\Delta U 1$ and the lower deviation $\Delta L 1$ of the operational dimension, to ensure that the concluding link of the process dimension chain (i.e., the corresponding design dimension obtained indirectly during machining ) finally meets the requirement of the design drawing(Wade 1967; Li and Li 2011; Martin et al. 2015; Wang et al. 2016).

The worst-case method of process dimension chain, which is used to calculate the operational dimension tolerance is too strict(Gao and Huang 2003; Hamou, et al. 2006; Li and Li 2011). In the process of operational dimension tolerance calculation, the machining error correlation(Sfantsikopoulos and Diplaris 1991; Hong and Chang 2002; Peng et al. 2008) and compensation effects (Chen 2011; Wang and Ren 2012; Heling et al. 2016; Hao et al. 2019) between the dimensions of each link in the process dimension chain are neglected. Under this condition, the operational dimension tolerance $D_{\text {Ope }}^{\Delta U 1} \underset{\Delta L 1}{\Delta}$ is too tight, i.e., implies extremely strict design parameters. If this tolerance is used to constrain the operational dimension during machining, while the other component links are actually qualified, then the operational dimension being qualified is a sufficient but not necessary condition for the design dimensionobtained indirectly to be qualified. In other words, if the operational dimension is 
qualified, the concluding link design dimension must be qualified. However, if the former is not qualified, the latter may be qualified, leading to the FR generation.

\subsection{Example 1.}

The design dimensions of the eccentric cylinder surface and the carburized layer of an eccentric shaft are shown in Fig. 2. To avoid clutter, other dimensions irrelevant to this study are omitted in the drawings.

It can be seen in Fig. 2 that the design dimensions of the two eccentric cylindrical surfaces are $\Phi$ $38_{-0.039}^{0}$ and $\Phi 46_{-0.05}^{0}$ respectively, and the carburized layer thickness on the eccentric cylinder $\Phi$ $38_{-0.039}^{0}$ is $0.5_{0}^{+0.3}$, so the design data of the carburized layer thickness is the cylindrical surface $\Phi$ $38_{-0.039}^{0}$.

During the actual machining of this eccentric shaft, the following steps are adopted to process the eccentric cylinders:

(1) Machining the cylindrical surface $\Phi 46_{-0.05}^{0}$, and semi-finish the cylindrical surface $\Phi 38_{-0.039}^{0}$ to dimension $D$.

(2) Carburizing and quenching the eccentric cylinder, and ensure the carburized layer thickness $0.5_{0}^{+0.3}$ to dimension $D_{\text {Ope }}$.

(2) Finish machining the eccentric cylindrical surface, directly ensure the dimension $\Phi 38_{-0.039}^{0}$ and concentricity $\Phi 0.06$, indirectly ensure the carburized layer thickness $0.5_{0}^{+0.3}$.

The operational dimension $D_{O p e}$ and data of the carburized layer of an eccentric shaft are shown in Fig. 3.

It can be seen in Fig. 3 that the carburized layer thickness assured directly is dimension $D_{\text {Ope }}$ during the actual processing, and its operational data is the cylindrical surface $D$. Therefore, $D_{\text {Ope }}$ is the operational dimension caused by the non-coincidence between the operational data and design data. Fig. 4 depicts the process dimension chain for the calculation of the operational dimension $D_{\text {Ope }}$.

In the process dimension chain, the design dimension $0.5_{0}^{+0.3}$ is the concluding link, while dimensions $\Phi 38_{-0.039}^{0}, D_{O p e}$ and $D$ correspond to the component links. The concentricity $\Phi 0.06$ can be converted into dimension $0 \pm 0.03$. This dimension can then be considered a component link(Tsai and Cutkosky 2009; Tong, et al. 2008). In this example, dimension $D$ is also an operational dimension, which differs from $D_{\text {Ope }}$. Dimension $D$ is caused by the machining allowance, not by the 
non-coincidence between the operational data and design data, so it needs to be determined by calculating the machining allowance, rather than calculating the process dimension chain. According to the eccentric cylinder machining allowance, the accuracy of the final dimension and semi-finishing cost-efficiency, dimension $D$ is determined to be $\Phi 38.4_{-0.150}^{0}$. To make each dimension in the process dimension chain end-to-end, the diameter dimensions $\Phi 38_{-0.039}^{0}$ and $\Phi 38.4_{-0.150}^{0}$ need to be converted into radii $19_{-0.0195}^{0}$ and $19.2_{-0.075}^{0}$.

The worst-case method is adopted to reversely calculate the process dimension chain(Wade 1967; Arora et al. 1995; Jaballi et al. 2009). Subsequently, the nominal value of the operational dimension $D_{\text {Ope }}$ is 0.7 , the upper deviation $\Delta U 1=+0.195$, and the lower deviation $\Delta L 1=+0.0495$, namely the operational dimension $D_{O p e}$ and its tolerance are $0.7_{+0.0495}^{+0.1950}$. This operational dimension tolerance calculated using the worst-case method of the process dimension chain is too tight. If this tolerance is used to constrain the operational dimension during machining, the operational dimension $D_{\text {Ope }}$ being qualified is a sufficient but not a necessary condition for the design dimension $0.5_{0}^{+0.3}$ to be qualified. In this case, if the operational dimension $D_{\text {Ope }}$ is not qualified, the design dimension $0.5_{0}^{+0.3}$ can still be qualified. For example, under the condition that dimensions $19_{-0.0195}^{0}$ and $19.2_{-0.075}^{0}$ are actually processed to 18.99 and 19.19 , respectively, and the actual error of the concentricity is 0.01 , when the actual value of the operational dimension $D_{\text {Ope }}$ is 0.95 , it is not within the tolerance of $0.7_{+0.0495}^{+0.1950}$, the maximum value of the concluding link is calculated as 0.76 . Considering the cylindrical shape of the carburized layer and symmetry of the concentricity tolerance(Mei 1996; Tong et al. 2008; Tsai and Cutkosky 2009), in the calculation of the actual value of carburized layer depth under the above conditions, the actual error of concentricity must also be considered as -0.01 . Then the minimum value of the concluding link is calculated as 0.74 . Therefore, the final actual range of carburized layer depth is indirectly assured $0.74 \sim 0.76$, which still is within a tolerance of $0.5_{0}^{+0.3}$. This implies that the design dimension $0.5_{0}^{+0.3}$, which is indirectly assured, may still be qualified under the condition that the operational dimension $D_{O p e}$ is not qualified. This is the FR caused by the disqualification of the operational dimension $D_{\text {Ope }}$.

\section{Judgment on the FR}

\subsection{The operational dimension tolerances causing the FR}

In the process dimension chain calculation, the operational dimension tolerance $D_{\text {Ope }}{ }^{\Delta U 1}$ c calculated 
by the worst-case method of the process dimension chain is too tight. By taking the operational dimension $D_{O p e}$ as the concluding link and using the design dimensions, which are obtained indirectly during machining as a component link, a new dimension chain is established. According to the judgment principle, the increasing and decreasing links need to be re-distinguished in this new dimension chain. The new dimension chain is positively calculated(Wade 1967; Jaballi et al. 2009; Li and Li 2011) using the worst-case method. Thus a new tolerance $D_{O p e}^{\Delta U 22} \underset{\Delta L 2}{\Delta}$ of the operational dimension is obtained. Compared with the tolerance $D_{O p e}^{\Delta U 1} \underset{1}{\Delta L 1}$ reversely calculated in the process dimension chain, the new tolerance $D_{O p e}^{\Delta U 2} \underset{\Delta L 2}{ }$ is relatively loose. Due to the mutual compensation relationship among the machining errors of the dimensions in this new dimension chain, for any actual value of the operational dimension within the new tolerance $D_{O p e}^{\Delta U 22} \underset{\Delta L 2}{ }$, the design dimension obtained indirectly can be finally qualified, if the machining tolerance of other dimensions is properly adjusted (Shah, et al. 1998; Hong and Chang 2003). If this new tolerance $D_{O p e \Delta L 2}^{\Delta U 2}$ is used to constrain the operational dimension during machining, the operational dimension $D_{\text {Ope }}$ being qualified becomes a necessary but not a sufficient condition for the design dimension to be qualified. In this case, two separated tolerances $D_{O p e}^{\Delta U 2} \underset{\Delta U 1}{\Delta}$ and $D_{O p e}^{\Delta L 1} \underset{\Delta L 2}{\Delta L^{2}}$ can be obtained by subtracting the above tighter

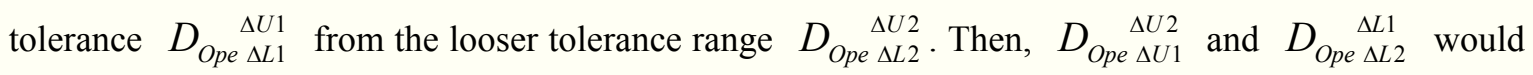
be the operational dimension tolerances causing the FR.

During machining, some dimensions in the new dimension chain are generated before the operational dimension generation, while others are generated afterward. All the increasing links in the new dimension chain are subdivided into dimensions $A_{p 1}$ and $A_{p 2}$, while the decreasing links are subdivided into dimensions $A_{q 1}$ and $A_{q 2}$. Of these, dimensions $A_{p l}$ and $A_{p 2}$ represent the increasing links formed before and after the operational dimension generation, respectively, while $A_{q 1}$ and $A_{q 2}$ denote the respective decreasing links. Because dimensions $A_{p l}$ and $A_{q l}$ are generated before the operational dimension generation, these dimensions' actual values can be obtained by measurement. Then the actual deviations of these dimensions can be used to replace their upper and lower deviations during the new dimension chain calculation. The operational dimension and its upper and lower deviations, which are positively calculated by the worst-case method in the new dimension chain, can be derived as follows:

$$
D_{\text {Ope }}=\sum A_{p 1}+\sum A_{p 2}-\sum A_{q 1}-\sum A_{q 2}
$$




$$
\begin{gathered}
\Delta U 2=\sum \Delta U\left(A_{p 2}\right)+\sum A_{p 1}^{*}-\sum \Delta L\left(A_{q 2}\right)-\sum A_{q 1}^{*} \\
\Delta L 2=\sum \Delta L\left(A_{p 2}\right)+\sum A_{p 1}^{*}-\sum \Delta U\left(A_{q 2}\right)-\sum A_{q 1}^{*}
\end{gathered}
$$

where $D_{\text {Ope }}$ is the operational dimension and the concluding link of the new dimension chain, $A^{*}$ is the actual deviation of dimension $A, \Delta U(A)$ is the upper deviation of dimension $A$, and $\Delta L(A)$ is the lower deviation of dimension $A$.

Now suppose that the dimension tolerance converted from geometric tolerance is involved in the new dimension chain calculation, and the error of this geometric tolerance can produce a compensation effect to ensure that the final value of the design dimension obtained indirectly is qualified(Tsai and Cutkosky 2009; Tong, et al. 2008). In this case, the dimension tolerance converted from this geometric tolerance can be directly applied to the new dimension chain calculation. Otherwise, the upper and lower deviations converted from this geometric tolerance must be considered zero in the new dimension chain calculation.

Under the condition that the actual deviations of dimensions $A_{p l}$ and $A_{p l}$ replace the upper and lower deviations during the new dimension chain calculation, the tolerance range $\delta_{2}^{*}$ of operational dimension can be derived from Eqs. (1) to (3) as follows:

$$
\delta_{2}^{*}=\Delta U 2-\Delta L 2=\sum \delta\left(A_{p 2}\right)+\sum \delta\left(A_{q 2}\right)
$$

where $\delta(A)$ is the tolerance range of dimension $A$.

If the upper and lower deviations of dimensions $A_{p l}$ and $A_{p l}$ are used to calculate the new dimension chain, the tolerance range $\delta_{2}$ of the operational dimension can be derived as follows:

$$
\delta_{2}=\sum \delta\left(A_{p 1}\right)+\sum \delta\left(A_{q 1}\right)+\sum \delta\left(A_{p 2}\right)+\sum \delta\left(A_{q 2}\right)
$$

Then, the following inequality can be obtained.

$$
\delta_{2}^{*}<\delta_{2}
$$

It can be concluded that for dimensions that were generated before the operational dimension, by using their actual deviation to replace the upper and lower deviation in the calculation of the new dimension chain, the calculated operational dimension tolerance that may produce FR is more accurate and more suitable for the actual situation of parts' processing.

The tolerances $D_{O p e \Delta U 1}^{\Delta U 2}$ and $D_{O p e \Delta L 2}^{\Delta L 1}$ causing the FR can be obtained by subtracting the tolerance $D_{O p e ~}^{\Delta U L 1}$ from the tolerance $D_{\text {Ope } \Delta L 2}^{\Delta U 2}$ derived from Eqs. (1) to (3).

Ultimately, the flowchart of operational dimension tolerances causing the FR and the process of 
judging the FR is presented in Fig. 5.

\subsection{Example 2.}

In the previous example, the operational dimension $D_{\text {Ope }}$ was determined as a concluding link in the new dimension chain. In the actual processing, the dimension $19.2_{-0.075}^{0}$ is generated before the operational dimension generation. In the new dimension chain calculation via Eqs.(2) and (3), the upper and lower deviations of the dimension $19.2_{-0.075}^{0}$ need to be replaced by the actual deviation. Under the condition that the actual value of the dimension $19.2_{-0.075}^{0}$ is 19.19 , its actual deviation is -0.01 . In this example, considering the carburized layer's cylinder shape and symmetry of the geometric tolerance, the carburized layer thickness in all directions of the circumference must be qualified in the actual processing process. The error of the concentricity tolerance is harmful and cannot produce a compensation effect to ensure that the final thickness of the carburized layer in all directions of the circumference is qualified. Then, the upper and lower deviations converted from the concentricity need to be regarded as zero in the new dimension chain calculation. The comparison of the calculation processes in both dimension chains is presented in Table 1.

Two separated tolerances $0.7_{+0.1950}^{+0.3095}$ and $0.7_{-0.0100}^{+0.0495}$ can be obtained by subtracting the smaller tolerance $0.7_{+0.0495}^{+0.1950}$ from the larger tolerance range $0.7_{-0.0100}^{+0.3095}$. Then, $0.7_{+0.1950}^{+0.3095}$ and $0.7_{-0.0100}^{+0.0495}$ are the operational dimension tolerances causing the FR. This implies that when the actual value of the dimension $19.2_{-0.075}^{0}$ is 19.18 , and the operational dimension is processed to $0.7_{+0.1950}^{+0.3095}$ and $0.7_{-0.0100}^{+0.0495}$, it does not meet the tolerances $0.7_{+0.0495}^{+0.1950}$, and the workpiece is classified as FR. Therefore, remedial measures should be taken in the subsequent processing of dimension $19_{-0.0195}^{0}$ and concentricity $\Phi 0.06$ to ensure that the final design dimension $0.5_{0}^{+0.3}$ is qualified.

\section{Remedial measures for the FR}

\subsection{Remedy method for the FR}

When the FR with an unqualified operational dimension occurs, it is necessary to adopt remedial measures in the subsequent processing based on the mutual compensation relationship among the component links' machining errors in the process dimension chain to avoid the real reject generation.

Under the condition that the operational dimension is the increasing link in the process dimension chain, the relationship between the actual deviations of each dimension should satisfy the following 
equation:

$$
A_{\Sigma}^{*}=\sum A_{p}^{*}+D_{O p e}^{*}-\sum A_{q}^{*}
$$

where $A_{\Sigma}^{*}$ is the actual deviation of the concluding link, $D_{O p e}^{*}$ is the actual deviation of the operational dimension, while $A_{p}^{*}$ and $A_{q}^{*}$ are the actual deviations of the increasing and decreasing links, respectively.

It follows from Eq.(7) that when the FR with the actual deviation $D_{\text {Ope }}^{*}$ of the operational dimension exceeding its upper deviation $\Delta U 1$ occurs, the actual values of other increasing links are required to be as small as possible within their tolerance, to keep the actual deviation $A_{\Sigma}^{*}$ of the design dimension $A_{\Sigma}$ as small as possible. In contrast, the actual values of the decreasing links have to be as large as possible. When the operational dimension is unqualified as the actual deviation $D_{O p e}^{*}$ is less than the lower deviation $\Delta L 1$, the actual values of the other increasing links should be as large as possible within their tolerance, in order to maximize the actual deviation of the design dimension $A_{\Sigma}$. In contrast, the actual values of the decreasing links should be as small as possible.

When the operational dimension is the decreasing link in the process dimension chain, the relationship between the actual deviations of each dimension can be expressed by the following formula:

$$
A_{\Sigma}^{*}=\sum A_{p}^{*}-\left(\sum A_{q}^{*}+D_{O p e}^{*}\right)
$$

According to Eq.(8), when the FR with the actual deviation $D_{O p e}^{*}$ of the operational dimension exceeding its upper deviation $\Delta U 1$ occurs, the actual values of the increasing link dimensions should be as large as possible, and the actual values of the other decreasing link dimensions should be as small as possible. When the operational dimension is unqualified as the actual deviation $D_{O p e}^{*}$ is less than the lower deviation $\Delta L 1$, the actual values of the increasing link dimensions should be as small as possible, and the actual values of the other decreasing link dimensions should be as large as possible.

\subsection{Calculation of the upper and lower deviations of the component link dimensions generated during the subsequent processing in the remediation of FR}

Suppose a particular dimension in the subsequent processing is required to be as large as possible in FR's remediation. In that case, the dimension's processing tolerance needs to be compressed to the upper deviation within the original tolerance. For dimension $A$, it is assumed that the upper deviation 
before tolerance compression is $\Delta U(A)$, the lower deviation is $\Delta L(A)$. The dimensional tolerance is $\delta(A)=\Delta U(A)-\Delta L(A)$. The upper deviation after tolerance compression to the upper deviation is still $\Delta U(A)$. At the same time, the lower deviation becomes $\Delta L^{\prime}(A)$, then the dimensional tolerance becomes $\delta^{\prime}(A)=\Delta U(A)-\Delta L^{\prime}(A)$. The tolerance compression amount of dimension $A$, which is the compensation amount $Q(A)$ of dimension $A$ to the operational dimension $D_{\text {Ope }}$, can be calculated as follows:

$$
Q(A)=\delta(A)-\delta^{\prime}(A)=\Delta L^{\prime}(A)-\Delta L(A)
$$

Suppose a particular dimension $B$ in the subsequent processing is required to be as small as possible. In that case, the following formula can be used to obtain the compensation amount $Q(B)$ of dimension $B$ to the operational dimension $D_{\text {Ope }}$ after tolerance compress to the lower deviation.

$$
Q(B)=\delta(B)-\delta^{\prime}(B)=\Delta U(B)-\Delta U^{\prime}(B)
$$

where $\Delta U(B)$ and $\Delta U^{\prime}(B)$ are the upper deviations of dimension $B$ before and after tolerance compression, respectively.

After the FR is produced, the difference between the actual deviation and the upper or lower deviation of the component link dimensions generated before the operational dimension generation has already provided the error compensation to the operational dimension. However, the remedial measures could not be applied to these dimensions in the subsequent processing. If dimension $C$ generated before the operational dimension generation needs to be as large as possible, the error compensation amount $R(C)$ provided by dimension $C$ to the operational dimension can be calculated as follows:

$$
R(C)=C^{*}-\Delta L(C)
$$

where $C^{*}$ is the actual deviation of dimension $C$, and $\Delta L(C)$ is the lower deviation of dimension $C$.

Similarly, if dimension $E$ generated before the operational dimension generation needs to be as small as possible, the error compensation amount $R(E)$ provided by dimension $E$ to the operational dimension is given by

$$
R(E)=\Delta U(E)-E^{*}
$$

where $\Delta U(E)$ is the upper deviation for dimension $E$, and $E^{*}$ is the actual deviation for dimension $E$.

In the remediation of $\mathrm{FR}$, the total compensation amount $\sum Q$ of the subsequent dimensions to the operational dimensions can be derived as

$$
\sum Q=W\left(D_{\text {ope }}\right)-\sum R
$$


where $W\left(D_{\text {Ope }}\right)$ is the overproof amount of the actual deviation $D_{\text {Ope }}^{*}$ of the operational dimension to the operational dimension tolerance $D_{\text {Ope }}^{\Delta U L 1}$. Noteworthy is that $W\left(D_{O p e}\right)=\Delta L 1-D_{O p e}^{*}$ if the actual deviation $D_{O p e}^{*}$ is less than the lower deviation $\Delta L 1$, while $W\left(D_{O p e}\right)=D_{O p e}^{*}-\Delta U 1$ if the actual deviation $D_{O p e}^{*}$ exceeds the upper deviation $\Delta U 1$. Here $\sum R$ is the total error compensation amount provided by the component link dimensions generated before the operational dimension generation, which can be calculated as follows:

$$
\sum R=\sum R(C)+\sum R(E)
$$

where $R(C)$ and $R(E)$ are derived via Eqs. (11) and (12), respectively.

Besides, $\Sigma Q$ has the following interrelations with the tolerance compression amount of each component link dimension generated in the subsequent processing obtained via Eqs. (9) and (10):

$$
\sum Q=\sum Q(A)+\sum Q(B)
$$

According to Eq.(13), the total tolerance compression amount $\sum Q$ of dimensions generated in the subsequent processing can be calculated and allocated to these dimensions. In allocating the tolerance compression amount, some factors,such as the original tolerance of each dimension and the processing manufacturability after tolerance compression, should be comprehensively considered.

After the total tolerance compression amount $\sum Q$ is allocated to dimensions generated in the subsequent processing, the upper or lower deviations of these dimensions after the tolerance compression can be determined. If the tolerance of dimension $A$ needs to be compressed to the upper deviation, and the tolerance compression amount assigned to dimension $A$ is $Q(A)$, according to Eq.(9), the lower deviation $\Delta L^{\prime}(A)$ after the tolerance compression can be derived as:

$$
\Delta L^{\prime}(A)=\Delta L(A)+Q(A)
$$

Similarly, suppose the tolerance of dimension $B$ needs to be compressed to the lower deviation, and the tolerance compression amount assigned to dimension $B$ is $Q(B)$, according to Eq.(10). In that case, the upper deviation $\Delta U^{\prime}(B)$ after the tolerance compression can be calculated as follows:

$$
\Delta U^{\prime}(B)=\Delta U(B)-Q(B)
$$

Based on Eqs. (13) to (17), the upper or lower deviations of the dimensions generated in the subsequent processing after tolerance compression can be obtained.

The flowchart of remedial measures using tolerance compression for FR's subsequent dimension is 
depicted in Fig. 6.

According to Eq.(13), after the FR occurrence, the difficulty of the remedy in the subsequent processing depends on the overproof amount $W\left(D_{\text {Ope }}\right)$ of the actual deviation $D_{\text {Ope }}^{*}$ to the operational dimension tolerance $D_{O p e} \underset{\Delta U 1}{\Delta U 1}$ and the total error compensation amount $\sum R$ provided by the component link dimensions generated before the operational dimension generation. Smaller values of $W\left(D_{\text {Ope }}\right)$ and larger values $\sum R$ results in a smaller total compensation amount $\sum Q$ required in the subsequent processing and in a less difficult remedy. If $\sum Q \leq 0$, then $W\left(D_{O p e}\right)$ is entirely compensated by the actual errors of the dimensions generated before the operational dimension generation. In the subsequent processing, the design dimension can be indirectly assured to be qualified without the dimensional tolerance compression.

\subsection{Examples of the FR remedy}

In the previous example, the operational dimension $D_{\text {Ope }}$ was an increasing link in the process dimension chain. Its tolerance was determined to be $0.7_{+0.0495}^{+0.1950}$ via the worst-case method. Because the dimension $19.2_{-0.075}^{0}$ was generated before the operational dimension generation, the remedial measures could not be taken to this dimension after FR's occurrence and had to be applied only to dimension $19_{-0.0195}^{0}$ and concentricity $\Phi 0.06$. The larger the concentricity error, the more difficult it is to ensure the final thickness of the carburized layer in all directions of the circumference is qualified, and concentricity is a symmetrical tolerance (Mei 1996; Tong, et al. 2008; Tsai and Cutkosky 2009). Therefore, the concentricity tolerance should be symmetrically compressed to zero in the process of the remedy for the FR. The concentricity tolerance before tolerance compression is $\Phi 0.06$. It is assumed that the concentricity tolerance after tolerance compression was $\Phi X$, and the tolerance compression amount assigned to the concentricity was $Q(X)$. The following formula is proposed.

$$
Q(X)=\frac{1}{2}(0.06-X)
$$

Then $X$ can be calculated as follows:

$$
X=0.06-2 Q(X)
$$

When the actual value of dimension $19.2_{-0.075}^{0}$ is 19.19 , the operational dimension tolerances causing the FR are $0.7_{+0.1950}^{+0.3095}$ and $0.7_{-0.0100}^{+0.0495}$. Under two different conditions, which imply that the actual values of the operational dimension $D_{\text {Ope }}$ are 1.00 (FR 1) and 0.70 (FR 2), respectively, the 
remedial measures in the subsequent processing and the calculation of the upper or lower deviation of dimension $19_{-0.0195}^{0}$ and concentricity $\Phi 0.06$ after tolerance compression were analyzed and summarized in Table 2.

Under the condition that the actual value of the operational dimension $D_{\text {Ope }}$ is 0.95 , the actual deviation of the operational dimension is $D_{\text {Ope }}^{*}=0.95-0.7=0.25$. Because $D_{O p e}^{*}>\Delta U 1, W\left(D_{O p e}\right)=D_{O p e^{-}}^{*}$ $\Delta U 1=0.25-0.195=0.055$. If the actual value of dimension $19.2_{-0.075}^{0}$ is 19.19 , its actual deviation is -0.01 , and the error compensation amount provided by this dimension is $\sum R=0.065$. Thus, the total compensation amount required for the subsequent dimension is $\sum Q=W\left(D_{\text {Ope }}\right)-\sum R=-0.01<0$. This implies that the overproof amount $W\left(D_{O p e}\right)$ of the actual deviation $D_{O p e}^{*}$ has been entirely compensated by the actual error of the dimension $19.2_{-0.075}^{0}$. Therefore, no tolerance compression to the dimensions $19_{-0.0195}^{0}$ and concentricity $\Phi 0.06$ is required anymore. In this case, it can be verified that if the remedial measure is not held in the subsequent process, the tolerance of the design dimension obtained indirectly is $0.5_{+0.2105}^{+0.2900}$, which meets the requirements of $0.5_{0}^{+0.3}$.

\section{Conclusions}

The above analysis of the fake reject(FR) occurrence cases with unqualified operational dimension made it possible to draw the following conclusions.

(1) It is shown that applying the worst-case method of process dimension chain to calculating the operational dimension tolerance yields too tight results. If this tolerance is used to constrain the operational dimension during machining, when the operational dimension is unqualified, the design dimension obtained indirectly can still be qualified. This is the fundamental cause of the FR of the operational dimension.

(2) The novel calculation method and flowchart of the operational dimension tolerance for judging the FR are proposed. The new dimension chain is established by treating the operational dimension as the concluding link. For dimensions generated before the generation of operational dimensions,their actual deviations are used to replace the upper and lower deviations in calculating this new dimension chain. Theoretical analysis and practical application results strongly indicate that the proposed method yields the operational dimension tolerance that may produce FR, which is accurate and more suitable for the actual parts' processing than available ones.

(3) Based on the mutual compensation relationship among the machining errors of the component 
links in the process dimension chain, the new FR's remedial measure by compressing dimension tolerance in subsequent processing is proposed. The proposed calculation method and flowchart of the upper or lower deviations and the tolerance of dimensions in subsequent processing after the tolerance compression are elaborated. This remedial measure can effectively avoid the FR being processed into real reject products in subsequent processing to enhance products' qualification rate.

(4) These paper findings can be applied to the treatment of process dimension FR in various cases. In this paper, the cylindrical surface's design dimensions and the carburized layer of an eccentric shaft are taken as an example for the method's feasibility verification. The process dimension chain calculation can be applied to all cases where the operational data do not coincide with the design one. FR's calculation and judgment method and the remedial measures on FR proposed in this paper apply to all operational dimensions calculated by the process dimension chain. Therefore, this study's results are considered instrumental in the FR treatment with non-coinciding operational and design data.

\section{Acknowledgments}

This work was supported by the National Natural Science Foundation of China (51805284) and the Basic and Frontier Technology Research Plan of Henan (152300410102).

\section{References}

Arora, V., Rao, P. N. and Rao, U. R. K. 1995. Dimension and tolerance specification for manufacturing planning. In: Conference Proceedings of Unigraphics users meet, New Delhi, 4.1-4.6.

Chen, S. 2011. Study on Reasons and Identification of False Waste Products in Machining. Journal of Chongqing Jiaotong University(Natural Science) 30(4): 885-888.

Gao, Y. and Huang, M. 2003. Optimal process tolerance balancing based on process capabilities. International Journal of Advanced Manufacturing Technology (21): 501-507.

Hao, J., Wang, Y. and Wang, X. 2019. Generalized definition and application of tolerance. Proceedings of the Institution of Mechanical Engineers, Part C: Journal of Mechanical Engineering Science 233(19-20): 6807-6814.

Hamou, S., Cheikh, A., Linares, J. M. and Daho, A. C. 2006. A stochastic concept for the optimization of manufacturing tolerances in computer aided process plan simulation. International Journal of 
Computer Integrated Manufacturing 19(7): 663-675.

Heling, B., Aschenbrenner, A., Walter, M. S. J. and Wartzack, S. 2016. On connected tolerances in statistical tolerance-cost-optimization of assemblies with interrelated dimension chains." Procedia CIRP (43): 262-267.

Hong, Y. S. and Chang, T. C. 2002. Tolerancing algebra: a building block for handling tolerance interactions in design and manufacturing part 1: concept and representation. International Journal of Production Research 40(18): 4633-4649.

Hong, Y. S. and Chang. T. C. 2003. Tolerancing algebra: a building block for handling tolerance interactions in design and manufacturing part 2: tolerance interaction. International Journal of Production Research 41(1): 47-63.

Hu, X. and Zhang, M. 2012. Research of a new method distinguishing false junks of workpieces. Machine Tool \& Hydraulics 40(22): 98-100.

Jaballi, K., Bellacicco, A., Louati, J., Rivière, A. and Haddar, M. 2009. Dimensioning of the intermediate states of the machined phases "DISMP" approach. International Journal of Advanced Manufacturing Technology 45(9):907-921.

Martin, A. J., Padmanaban, K. P. and Thiagarajan, K. 2015. Manufacturing tolerance design based on fuzzy binary approach. Transactions of the Canadian Society for Mechanical Engineering, 39(1): 85-95.

Mei, J. 1996. Tolerance analysis for setup planning in computer-aided process planning. Doctoral Thesis, Texas Tech University.

Li, S., Chen X., Dong B. 2012. Research and improvement measures of false waste area in process size when data does not coincide. Coal Mine Machinery 38(11): 112-114.

Li, Y. 2005. Research on the ambiguous rejects in technological process. Journal of Henan University of Technology (Natural Science Edition) 26(2): 58-60.

Li, Y., Li, W. and Ge, Z. 2011a. Research of Optimizing Cost Model of Tolerance Based on Robust Design. Information Japan 15(12). https://doi.org/10.1109/ICQR2MSE.2011.5976562. 
Li, Y. and Li, W. 2011b. Robust design of dimension chain with less component links and higher precision requirement. In International Conference on Quality. IEEE. https://doi.org/10. 1109/ICQR2MSE.2011.5976737.

Liu, L. 2015. Product research-tolerance machining process in terms of the size. Manufacturing Technology \& Machine Tool (8): 78-81.

Peng, H. P., Jiang, X. Q. and Liu, X. J. 2008. Concurrent optimal allocation of design and process tolerances for mechanical assemblies with interrelated dimension chains. International Journal of Production Research 46(24): 6963-6979.

Sfantsikopoulos, M. M. 1990. A cost-tolerance analytical approach for design and manufacturing. International Journal of Advanced Manufacturing Technology (5): 126-134.

Sfantsikopoulos, M. M. and Diplaris, S. C. 1991. Coordinate tolerancing in design and manufacturing. Robotics and Computer-Integrated Manufacturing 8(4): 219-222.

Shah, J. J., Yan, Y. and Zhang, B. C. 1998. Dimension and tolerance modeling and transformations in feature based design and manufacturing. Journal of Intelligent Manufacturing 9(5): 475-488.

Tong, C., Huang, J. and Dong, S. 2008. How considering geometrical tolerancing in calculment of dimension chain__ application of tolerance principles in calculment of dimension chain. Modern Manufacturing Engineering (1):89-91.

Tsai, J. C. and Cutkosky, M. R. 2009. Representation and reasoning of geometric tolerances in design. Artificial Intelligence for Engineering Design Analysis \& Manufacturing 11: 325-341.

Wade, O. R. 1967. Tolerance control in design and manufacturing. New York: Industrial Press.

Wang, X. and Ren, S. 2012. Research on theory and applications of virtual tolerance. Transactions of the Canadian Society for Mechanical Engineering 36(3): 233-240.

Wang, X., Sun, C. and Chen, J. 2013. Extension and application of the range of tolerance. Transactions of Beijing Institute of Technology 33(12): 1243-1246+1288.

Wang, X., Sun C., Yao, Y. and Liang, L. 2014. Extension of the definition of tolerance and an application thereof in the calculation of dimension chains. International Journal of Advanced 
Manufacturing Technology 71(5-8): 1069-1076.

Wang, X., Guo S., Che D. and Lan, G. 2019. Theory and application of virtual tolerance based on set concept. Journal of Mechanical Engineering 55(7): 172-177.

Wang, Y., Wang, X., Zhang, X. and Dai, L. 2016. Establishment and application of a process dimension tree. International Journal of Production Research 54(15): 4658-4668.

Zhang, H. C., Huang, S. H. and Mei, J. 1996. Operational dimensioning and tolerancing in process planning: setup planning. International Journal of Production Research 34(7): 1841-1858.

\section{Tables and figure captions}

Table 1.Comparison of calculation processes in both dimension chains

Table 2. Analysis of remedial measures for the fake reject (FR) in two cases

Fig. 1. The present study's flowchart

Fig. 2. Design dimensions of eccentric cylindrical surface and the carburized layer of an eccentric shaft Fig. 3. Operational dimensions and data of the carburized layer of an eccentric shaft

Fig. 4. Process dimension chain diagram of processing the carburized layer of an eccentric shaft Fig. 5. Flowchart of the FR identification

Fig. 6. The flowchart of remedial measures using tolerance compression for FR's subsequent dimension: calculation of the upper or lower deviations of subsequent dimension after tolerance compression 


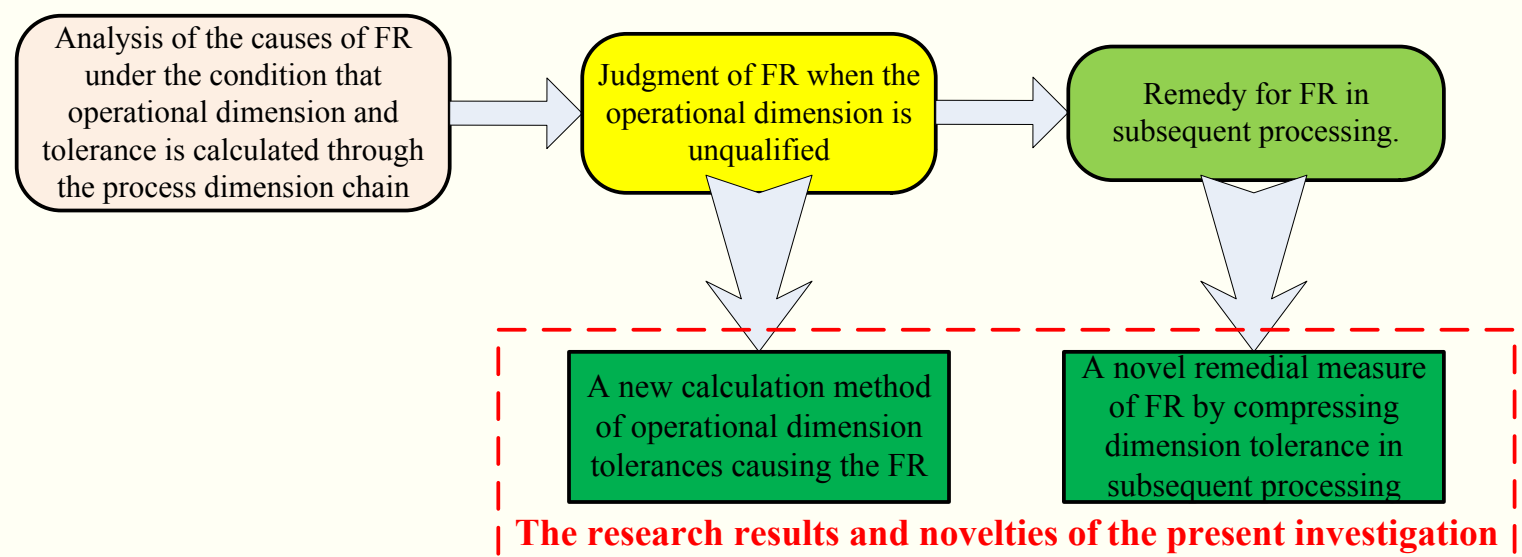

Fig. 1. The present study's flowchart

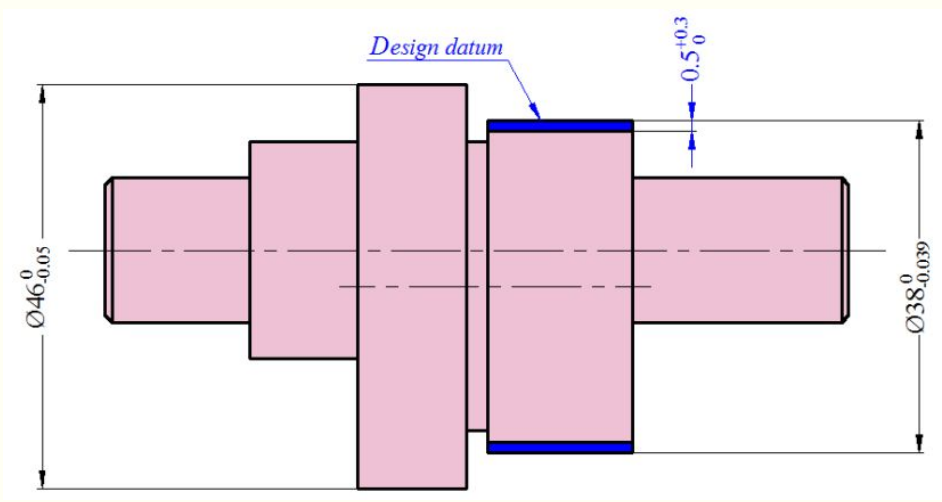

Fig. 2. Design dimensions of eccentric cylindrical surface and the carburized layer of an eccentric shaft

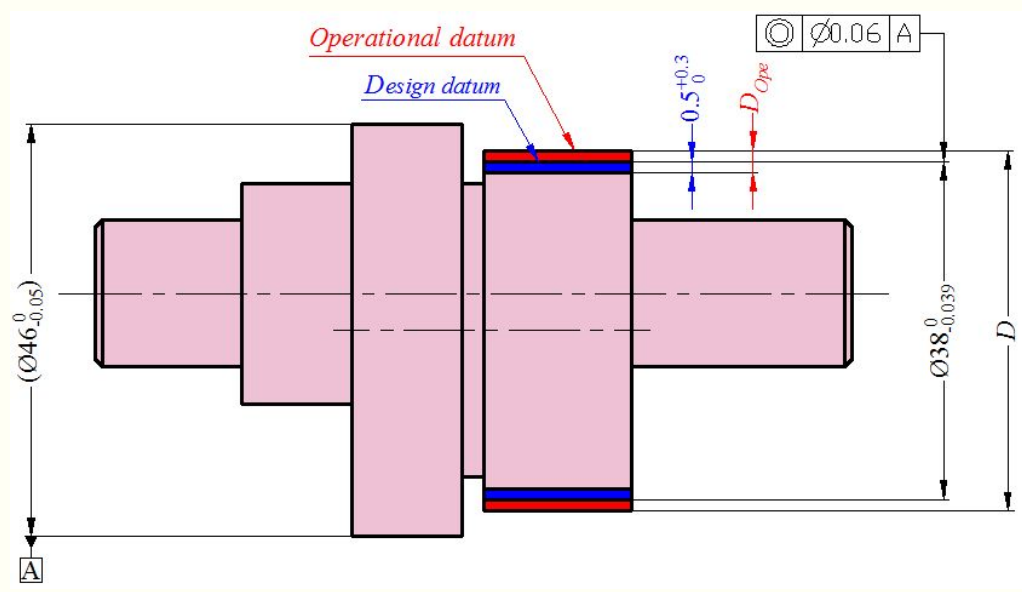

Fig. 3. Operational dimensions and data of the carburized layer of an eccentric shaft 


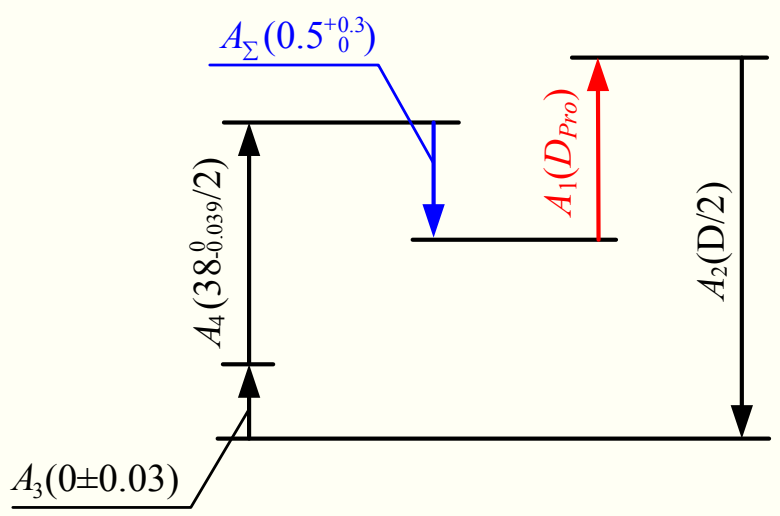

Fig. 4. Process dimension chain diagram of processing the carburized layer of an eccentric shaft

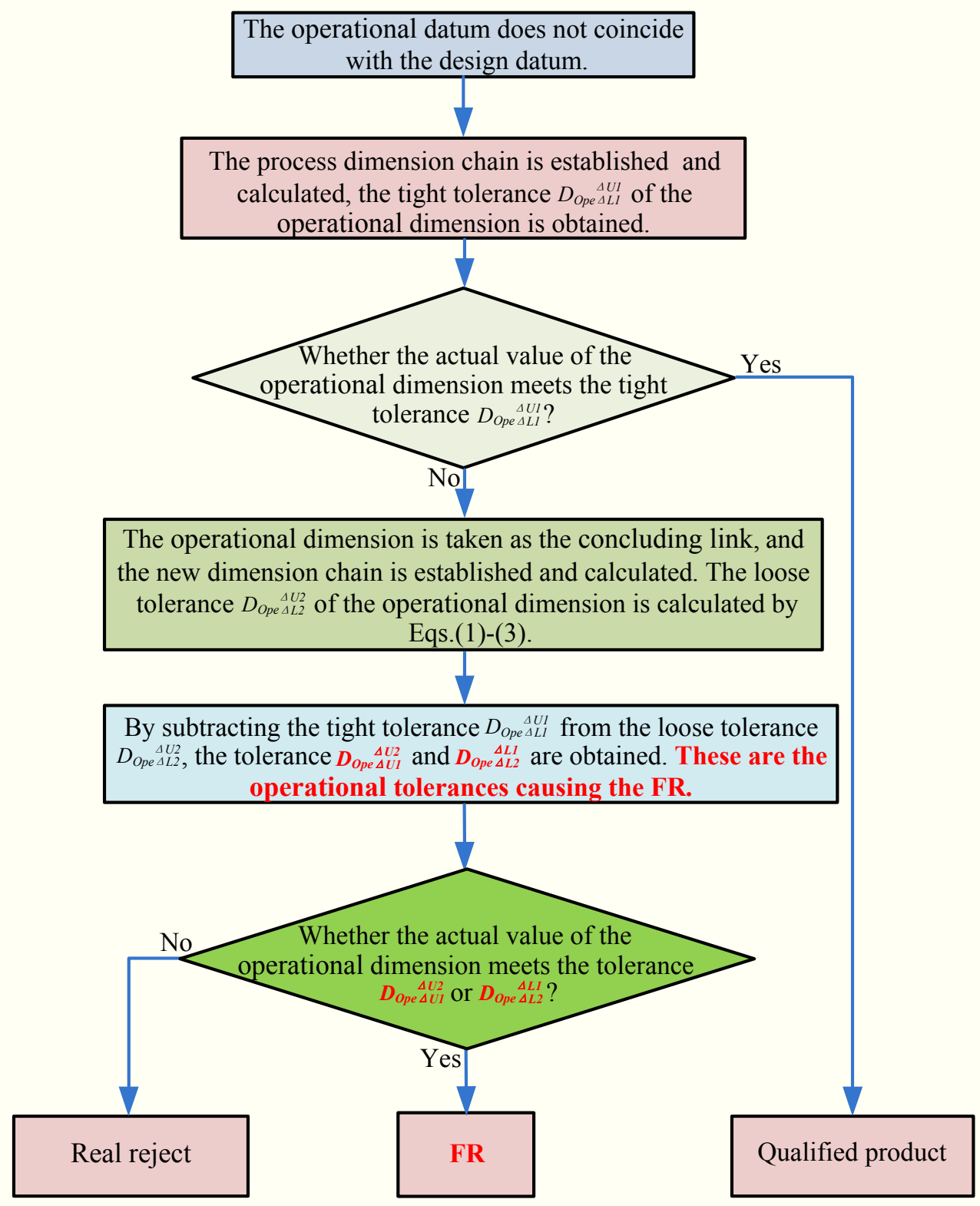

Fig. 5. Flowchart of the FR identification 


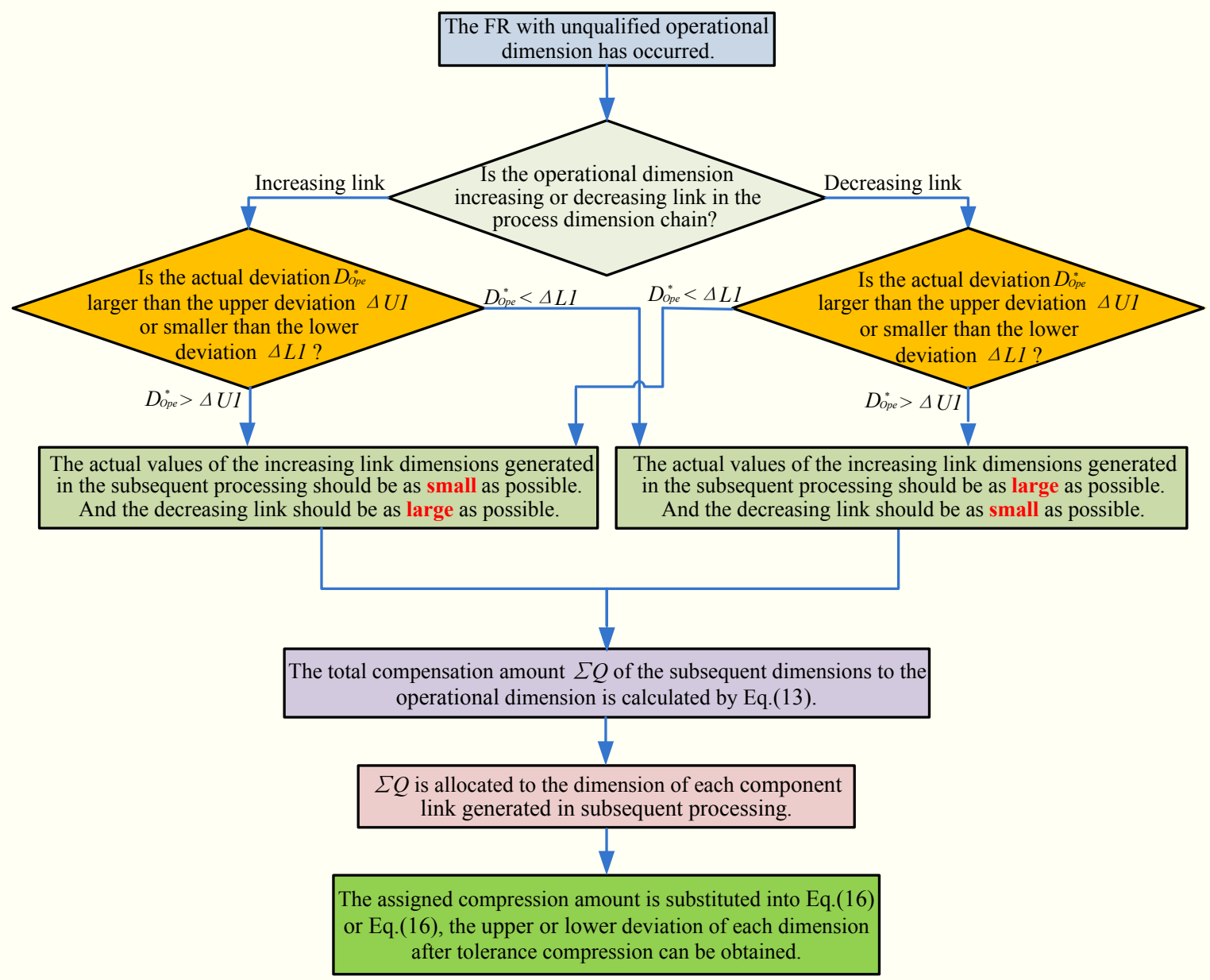

Fig. 6. The flowchart of remedial measures using tolerance compression for FR's subsequent dimension: calculation of the upper or lower deviations of subsequent dimension after tolerance compression 
Table 1. Comparison of calculation processes in both dimension chains

\begin{tabular}{|c|c|c|c|}
\hline & & Process dimension chain & New dimension chain \\
\hline \multirow[t]{2}{*}{$19_{-0.0195}^{0}$} & $\begin{array}{l}\text { Increasing link or } \\
\text { Decreasing link? }\end{array}$ & Increasing link & Decreasing link \\
\hline & Deviation & 0 and -0.0195 & 0 and -0.0195 \\
\hline \multirow[t]{2}{*}{$19.2_{-0.075}^{0}$} & $\begin{array}{l}\text { Increasing link or } \\
\text { Decreasing link? }\end{array}$ & Decreasing link & Increasing link \\
\hline & Deviation & 0 and -0.075 & -0.01 \\
\hline \multirow{2}{*}{$\begin{array}{l}\text { Concentricity } \\
\Phi 0.06\end{array}$} & $\begin{array}{l}\text { Increasing link or } \\
\text { Decreasing link? }\end{array}$ & Increasing link & Decreasing link \\
\hline & Deviation & +0.03 and -0.03 & 0 \\
\hline \multirow[t]{2}{*}{$0.5_{0}^{+0.3}$} & $\begin{array}{l}\text { Increasing link or } \\
\text { Decreasing link? }\end{array}$ & Concluding link & Increasing link \\
\hline & Deviation & +0.3 and 0 & +0.3 and 0 \\
\hline \multirow{4}{*}{$\begin{array}{l}\text { Operational } \\
\text { dimension } D\end{array}$} & $\begin{array}{l}\text { Increasing link or } \\
\text { Decreasing link? }\end{array}$ & Increasing link & Concluding link \\
\hline & $\begin{array}{l}\text { Calculation } \\
\text { method }\end{array}$ & $\begin{array}{l}\text { Worst case method reversely } \\
\text { calculated }\end{array}$ & $\begin{array}{l}\text { Worst case method positively } \\
\text { calculated }\end{array}$ \\
\hline & Result & $0.7_{+0.0495}^{+0.1950}$ & $0.7_{-0.0100}^{+0.3095}$ \\
\hline & Result analysis & $\begin{array}{l}\text { The sufficient and unnecessary } \\
\text { conditions for the blueprint } \\
\text { dimension being qualified }\end{array}$ & $\begin{array}{c}\text { The necessary and insufficient } \\
\text { conditions for the blueprint } \\
\text { dimension being qualified }\end{array}$ \\
\hline
\end{tabular}


Table 2. Analysis of remedial measures for the fake reject (FR) in two cases

\begin{tabular}{|c|c|c|c|}
\hline & & FR 1 & FR 2 \\
\hline \multirow{5}{*}{$\begin{array}{c}D_{\text {Ope }} \\
0.7_{+0.0495}^{+0.1950}\end{array}$} & $\begin{array}{c}\text { Increasing link or Decreasing } \\
\text { link? }\end{array}$ & Increasing link & Increasing link \\
\hline & Actual value & 1.00 & 0.70 \\
\hline & Actual deviation $D_{O p e}^{*}$ & 0.3 & 0 \\
\hline & $D_{O p e}^{*}>\Delta U 1$ or $D_{O p e}^{*}<\Delta L 1 ?$ & $D_{O p e}^{*}>\Delta U 1$ & $D_{O p e}^{*}<\Delta L 1$ \\
\hline & $W\left(D_{O p e}\right)$ & $D_{O p e^{-}-\Delta U 1=0.105}^{*}$ & $\Delta L 1-D_{O p e}^{*}=0.0495$ \\
\hline \multirow{5}{*}{$19.2_{-0.075}^{0}$} & $\begin{array}{c}\text { Increasing link or Decreasing } \\
\text { link? }\end{array}$ & Decreasing link & Decreasing link \\
\hline & $\begin{array}{l}\text { Requirements of remedial measures } \\
\text { for this dimension }\end{array}$ & $\begin{array}{l}\text { The actual value is as } \\
\text { large as possible. }\end{array}$ & $\begin{array}{l}\text { The actual value is as } \\
\text { small as possible. }\end{array}$ \\
\hline & Actual value & 19.19 & 19.19 \\
\hline & Actual deviation & -0.01 & -0.01 \\
\hline & $\begin{array}{c}\text { Error compensation amount generated } \\
\text { by the actual deviation of this } \\
\text { dimension }\end{array}$ & $\begin{array}{l}\text { Calculated by Eq.(11), } \\
\qquad 0.065\end{array}$ & $\begin{array}{l}\text { Calculated by Eq.(12), } \\
0.01\end{array}$ \\
\hline & $\sum R$ & 0.065 & 0.01 \\
\hline & $\sum Q$ & $W\left(D_{\text {Ope }}\right)-\sum R=0.04$ & $W\left(D_{\text {Ope }}\right)-\sum R=0.0395$ \\
\hline \multirow{5}{*}{$19_{-0.0195}^{0}$} & $\begin{array}{c}\text { Increasing link or Decreasing } \\
\text { link? }\end{array}$ & Increasing link & Increasing link \\
\hline & $\begin{array}{l}\text { Requirements of remedial measures } \\
\text { for this dimension }\end{array}$ & $\begin{array}{l}\text { The actual value is as } \\
\text { small as possible. }\end{array}$ & $\begin{array}{l}\text { The actual value is as } \\
\text { large as possible. }\end{array}$ \\
\hline & Tolerance compression amount & 0.015 & 0.0145 \\
\hline & $\begin{array}{l}\text { Variation of upper or lower deviation } \\
\text { after tolerance compression }\end{array}$ & $\begin{array}{l}\text { Via Eq.(17), the upper } \\
\text { deviation is }-0.015 \text {. }\end{array}$ & $\begin{array}{l}\text { Via Eq.(16), the lower } \\
\text { deviation is }-0.005 \text {. }\end{array}$ \\
\hline & $\begin{array}{l}\text { Dimensional tolerance after } \\
\text { compression }\end{array}$ & $19_{-0.0195}^{-0.0150}$ & $19_{-0.005}^{0}$ \\
\hline \multirow{3}{*}{$\begin{array}{l}\text { Concentricity } \\
\Phi 0.06\end{array}$} & $\begin{array}{l}\text { Requirements of remedial measures } \\
\text { for this dimension }\end{array}$ & $\begin{array}{l}\text { The actual value is as } \\
\text { close to } 0 \text { as possible. }\end{array}$ & $\begin{array}{l}\text { The actual value is as } \\
\text { close to } 0 \text { as possible. }\end{array}$ \\
\hline & Tolerance compression amount $Q(X)$ & 0.025 & 0.025 \\
\hline & $\begin{array}{l}\text { Concentricity tolerance after } \\
\text { tolerance compression }\end{array}$ & $\begin{array}{l}\text { Via Eq.(19), the } \\
\text { concentricity tolerance is } \\
\qquad 0.01\end{array}$ & $\begin{array}{l}\text { Via Eq.(19), the } \\
\text { concentricity tolerance } \\
\text { is } \Phi 0.01\end{array}$ \\
\hline \multirow[b]{2}{*}{$0.5_{0}^{+0.3}$} & Dimension type & Concluding link & Concluding link \\
\hline & $\begin{array}{c}\text { Dimensional tolerance obtained } \\
\text { indirectly after taking remedial } \\
\text { measures }\end{array}$ & $\begin{array}{c}0.5_{+0.2855}^{+0.3000} \text {, which meets } \\
\text { the requirements of } \\
\text { tolerance } 0.5_{0}^{+0.3}\end{array}$ & $\begin{array}{l}0.5_{0}^{+0.015} \text {, which } \\
\text { meets the requirements } \\
\text { of tolerance } 0.5_{0}^{+0.3}\end{array}$ \\
\hline
\end{tabular}

